\title{
The Location of Persistent Amphetamine-Induced Changes in the Density of Dendritic Spines on Medium Spiny Neurons in the Nucleus Accumbens and Caudate-Putamen
}

\author{
Yilin Li', Bryan Kolb ${ }^{2}$ and Terry E Robinson*,I \\ 'Department of Psychology and Neuroscience Program, The University of Michigan, Ann Arbor, MI, USA; ${ }^{2}$ Canadian Center for Behavioral \\ Neuroscience, University of Lethbridge, Lethbridge, $A B$, Canada
}

\begin{abstract}
Repeated intermittent treatment with amphetamine increases the density of dendritic spines on medium spiny neurons (MSNs) in the nucleus accumbens (NAcc). The purpose of this study was two-fold: (I) to determine whether amphetamine has similar effects on MSNs in the caudate-putamen ( $\mathrm{CPu}$ ) and (2) to determine if this effect is localized to distal dendrites, the site of convergence of dopamine (DA) and glutamate synapses in the striatum. An amphetamine treatment regimen that produced behavioral sensitization increased the density of dendritic spines on MSNs in both the NAcc and dorsolateral CPu. This effect was long lasting, because it was evident 3.5 months after the discontinuation of drug treatment. The increase in spine density was confined to distal dendrites of MSNs, and was not apparent on dendrites close to the cell body. It is concluded that amphetamine may preferentially reorganize synapses at the site of DAglutamate interaction in the striatum, which may alter DA-glutamate signaling and thereby contribute to some of the persistent behavioral and psychological consequences of repeated exposure to psychostimulant drugs.

Neuropsychopharmacology (2003) 28, 1082-1085, advance online publication, 26 March 2003; doi:I 0. I038/sj.npp. I 3001 I 5
\end{abstract}

Keywords: sensitization; amphetamine; dendrites; neuroplasticity

\section{INTRODUCTION}

Repeated exposure to drugs of abuse can produce many long-lasting changes in brain and behavior, some of which may contribute to the development of psychopathological states, including psychosis and addiction (Robinson and Becker, 1986; Robinson and Berridge, 2000; Segal et al, 1981). There has been, therefore, considerable interest in identifying the nature of persistent drug-induced changes in neural circuits implicated in drug-associated psychopathology. One form of drug-induced synaptic plasticity involves alterations in patterns of dendritic branching and the density of dendritic spines in the nucleus accumbens (NAcc). Specifically, repeated treatment with amphetamine, cocaine, nicotine, or morphine alters the density of dendritic spines on the distal dendrites of medium spiny neurons (MSNs) in the NAcc, which presumably reflects a reorganization of synapses on these cells (Brown and Kolb, 2001; Robinson and Kolb, 1997, 1999a, b).

*Correspondence: Dr TE Robinson, Department of Psychology (Biopsychology), University of Michigan, 525 East University, Ann Arbor, MI 48I09-II09, USA, Fax: +I 734963 7480, E-mail: ter@umich.edu

Received 05 September 2002; revised 18 October 2002; accepted 30 October 2002

Online publication: 04 November 2002 at http://www.acnp.org/ Citations/Npp I 10402425
The exact locus of psychostimulant drug-induced changes in spine density on MSNs is of interest because different portions of the dendritic tree of these cells receive different inputs. The distal dendrites of MSNs are innervated primarily by projections arising extrinsic to the striatum, such as dopamine (DA) projections from the midbrain and glutamate projections from cortical structures (including the hippocampus and amygdala). Dendrites more proximal to the soma are innervated primarily by inputs of intrinsic origin (Groenewegen et al, 1991; Smith and Bolam, 1990). Furthermore, both DA and glutamate have been implicated in one form of neurobehavioral plasticity associated with repeated psychostimulant drug treatment, behavioral sensitization (Vanderschuren and Kalivas, 2000; Wolf, 1998). Thus, to the extent that psychostimulant drug-induced alterations in DA/glutamate signaling are related to psychostimulant drug-induced changes in the density of spines on MSNs, and to behavioral sensitization, we predicted that drug-induced changes in spine density might be confined to the distal dendrites of MSNs. However, the effect of psychostimulant drugs on distal $v s$ proximal dendrites of MSNs has never been directly examined, nor has it been determined whether similar effects are found on MSNs in both the caudate-putamen $(\mathrm{CPu})$ and the NAcc. The purpose of the present study, therefore, was to compare the effect of repeated treatment with amphetamine on the density of dendritic spines on both distal and proximal 
dendrites of MSNs in both the NAcc and the dorsolateral $\mathrm{CPu}$.

\section{METHODS}

Adult female Sprague-Dawley rats that were initially housed singly received 20 consecutive daily injections (i.p.) of $d$ amphetamine sulfate $(N=8)$ or saline $(N=6)$. The first and last amphetamine injections consisted of $0.5 \mathrm{mg} / \mathrm{kg}$, and the remaining 18 injections were $4.0 \mathrm{mg} / \mathrm{kg}$ (weight of the salt dissolved in saline). Locomotor activity (cage crossovers) was measured on the first and the last day of treatment. After the last treatment, the rats were housed in groups of three in $40.6 \times 24.1 \times 17.9 \mathrm{~cm}^{3}$ high stainless-steel hanging cages for 3.5 months. They were then anesthetized with an overdose of sodium pentobarbital and perfused through the heart with $0.9 \%$ saline. The brains were removed and placed in vials containing Golgi-Cox solution. The brains were cut into $200 \mu \mathrm{m}$ coronal sections using a vibrating microtome and stained using procedures described previously (Gibb and Kolb, 1998).

A Leica Model DMRE microscope equipped with a Ludl $\mathrm{XYZ}$ motorized stage was used at low power $(\times 100-250)$ to identify MSNs in the shell of the NAcc and the dorsolateral quadrant of the $\mathrm{CPu}$ for analysis. In order to be included in the analysis the extent of the dendritic tree of a cell had to be well stained and visible, and not be obscured by blood vessels, astrocytes, or other cells. Given the small number of cells in the NAcc and CPu that were stained essentially every cell that met these criteria was included in the analysis. Neuroleucida ${ }^{\circledR}$ (Microbrightfield, Inc.) software was then used to trace dendritic segments and count spines on selected dendrites. The program then calculated the actual length of the dendritic segment sampled and determined the number of spines visible per $10 \mu \mathrm{m}$ of dendrite. For this an oil immersion objective with a total magnification of $\times 1600$ was used, and with additional magnification from the video signal the final magnification on the video screen was approximately $\times 4000$. Two different portions of the dendritic tree were examined. Distal dendrites were terminal dendrites (third order or greater) and spines were always counted from the last branch point to the terminal tip of the dendrite. For proximal dendrites spines were counted beginning $17 \mu \mathrm{m}$ after spines first appeared distal to the soma, for an additional $30 \mu \mathrm{m}$ (see Comery et al, 1995). These were 2-3 order dendrites, but never terminal dendrites. The density of spines on both distal and proximal dendrites was determined for at least five cells per hemisphere, and no more than one proximal and one distal dendritic segment was sampled from any one cell. Thus, for each group distal and proximal dendritic segments were sampled from a total of 60-89 cells. Values from cells within a hemisphere were averaged and to test for hemispheric asymmetries, hemisphere was used as a factor in the analysis. All slides were coded such that the person conducting the anatomical analysis was blind to the treatment condition.

The behavioral data were analyzed using a paired $t$-test, and the spine data were analyzed using two-way analyses of variance, with treatment (saline vs amphetamine) and hemisphere (left $v s$ right) as the two factors.

\section{RESULTS AND DISCUSSION}

As expected, the amphetamine treatment protocol produced behavioral sensitization. The mean $( \pm$ SEM) number of cage crossovers following the last injection of amphetamine $(72.0 \pm 9.1)$ was significantly higher than following the first $(44.8 \pm 6.8 ; t=2.96, p<0.01)$.

Figure 1 shows a representative MSN in the NAcc and the insets show examples of distal and proximal dendritic segments. Figure 2 (top) shows that amphetamine treatment significantly increased the density of dendritic spines on the distal dendrites of MSNs in both the NAcc (effect of treatment, $\mathrm{F}=73.5, p<0.0001$; effect of hemisphere and interaction not significant, F's $<1.0$ ) and the CPu (effect of treatment, $\mathrm{F}=4.1, p=0.05$; effect of hemisphere and interaction not significant, F's $<1.0$ ). However, there was no effect of amphetamine treatment on the density of dendritic spines on proximal dendrites of MSNs in either the NAcc $(\mathrm{F}=0.8, p=0.4)$ or $\mathrm{CPu}(\mathrm{F}=2.5, p=0.13)$. The number of spines with multiple heads (branched spines) was also quantified. Amphetamine treatment significantly increased the number of branched spines on distal dendrites of MSNs in the NAcc (saline, 0.431 \pm 0.025 ; amphetamine, $0.588 \pm 0.032$; effect of treatment, $\mathrm{F}=12.1$, $p<0.002$; effect of hemisphere and interaction not significant, F's $<0.1$ ), but had no effect on branched spines on distal dendrites in the $\mathrm{CPu}(\mathrm{F}=0.3)$, or on proximal dendrites in either the NAcc or $\mathrm{CPu}\left(\mathrm{F}^{\prime} \mathrm{s}=1.4\right.$ and 0.5 , respectively).

The observation that amphetamine increases spine density on distal but not proximal dendrites of MSNs is consistent with an earlier report that amphetamine and cocaine increase the number of distal but not proximal dendritic branches on NAcc MSNs (Robinson and Kolb, 1999a). The precise localization of amphetamine-induced changes in the structure of dendrites on MSNs is of special

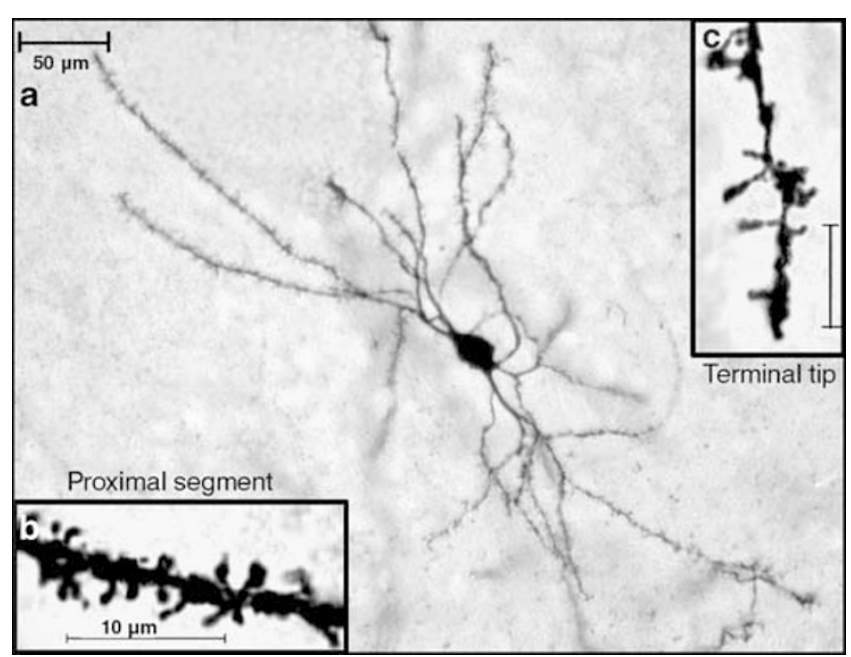

Figure I Photomicrograph of a representative Golgi-stained MSN in the NAcc (a). Four pictures were taken at different Z-levels and merged to enhance the portion of the dendritic tree in focus (the scale for this image is in the upper left corner). The insets show an example of a proximal dendritic segment (b) and the terminal tip of a distal dendrite (c) at approximately the same level of magnification at which spines were counted (scale bar in inset). 


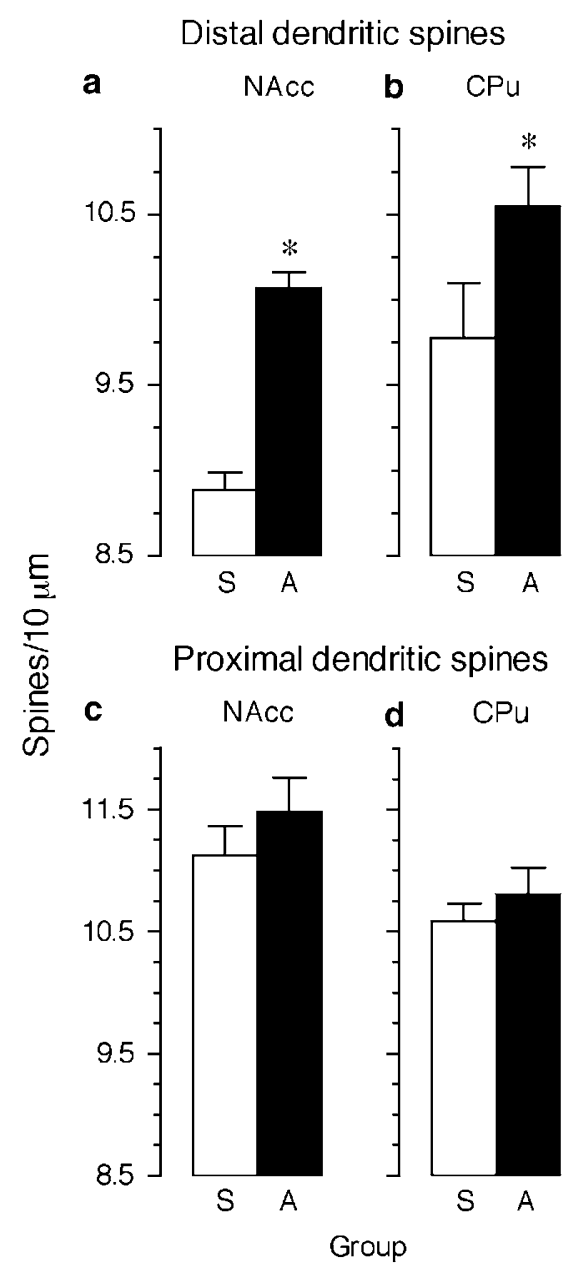

Figure 2 Mean (+SEM) number of dendritic spines on the distal (top) or proximal (bottom) dendrites of MSNs in the shell of the NAcc or the dorsolateral quadrant of CPu of rats treated 3.5 months earlier with either saline (S) or amphetamine (A). There was no significant effect of hemisphere (see text) and therefore to simplify the graphs the data were collapsed across hemisphere. *Indicates significantly different from the saline group.

interest because this suggests that repeated exposure to amphetamine produces a persistent reorganization of very specific synaptic inputs onto MSNs. Spines on the distal dendrites of MSNs receive inputs primarily from two sources that are extrinsic to the striatum. The heads of these spines are innervated by boutons that form an asymmetric synapse that is thought to consist of a glutamate input from sensory-motor cortex in the case of the dorsolateral $\mathrm{CPu}$, and from the prefrontal cortex and hippocampus in the case of the NAcc. Many of these same spines receive a DA input that forms a symmetric synapse on the neck of the spine or the nearby shaft of the dendrite. In contrast, more proximal portions of the dendritic tree of MSNs receive inputs of intrinsic origin (Groenewegen et al, 1991; Smith and Bolam, 1990). Thus, the present study suggests that amphetamine induces a reorganization of synapses specifically involved in the integration of DA and glutamate signaling in the striatum, although detailed ultrastructural studies will be required to test this hypothesis.
Of course, both DA and glutamate have been prominently implicated in the induction and the expression of behavioral sensitization (Vanderschuren and Kalivas, 2000; Wolf, 1998). Therefore, the persistent reorganization of synapses at the specific site of convergence of DA and glutamate signaling on MSNs may represent the structural basis for persistent sensitization-related changes in responsiveness to psychomotor stimulant drugs and other stimuli-changes that are thought to contribute to the compulsive patterns of drug-seeking and drug-taking behavior that characterize addiction (Robinson and Berridge, 2000, 2003).

In recent years, research on drug experience-dependent plasticity has focused to a large extent on the NAcc, but the comparison of the dorsal and ventral striatum reported here also serves to remind us that drugs of abuse have persistent effects on more than the NAcc. For example, many early studies on the neurobiology of sensitization found that repeated treatment with psychostimulant drugs increases evoked DA release from striatal slices, which consisted primarily of $\mathrm{CPu}$ (Kolta et al, 1985; Robinson and Becker, 1982), and later microdialysis studies showed increased DA release in both the NAcc and CPu (Kazahaya et al, 1989; Paulson and Robinson, 1995). Indeed, repeated treatment with drugs of abuse causes neuroadaptations in many brain regions (Robinson and Berridge, 2000; Vanderschuren and Kalivas, 2000), which presumably account for the diversity of behaviors and psychological processes that are altered by these drugs. Thus, the structural changes in the NAcc reported here may be related to alterations in functions associated with this structure, such as incentive motivational processes or drug-induced locomotor activity, whereas changes in the $\mathrm{CPu}$ may be related to alterations in drug-induced stereotyped behavior or habit learning, which have been associated with the $\mathrm{CPu}$. However, to determine exactly what changes at what synapses in what structures mediate what changes in behavior remains a formidable challenge.

\section{ACKNOWLEDGMENTS}

This research was funded by a grant from NIDA (R01 DA13398), and TER was supported by a Senior Research Scientist Award from NIDA (K05 DA00473).

\section{REFERENCES}

Brown RW, Kolb B (2001). Nicotine sensitization increases dendritic length and spine density in the nucleus accumbens and cingulate cortex. Brain Res 899: 94-100.

Comery TA, Shah R, Greenough WT (1995). Differential rearing alters spine density on medium-sized spiny neurons in the rat corpus striatum: evidence for association of morphological plasticity with early response gene expression. Neurobiol Learn Mem 63: 217-219.

Gibb R, Kolb B (1998). A method for vibratome sectioning of Golgi-Cox stained whole rat brain. J Neurosci Methods 79: $1-4$.

Groenewegen HJ, Berendse HW, Meredith GE, Haber SN, Voorn P, Wolters JG et al (1991). Functional anatomy of the ventral, limbic system-innervated striatum. In: Willner P, Scheel-Krüger 
J (eds). The Mesolimbic Dopamine System: From Motivation to Action. John Wiley \& Sons: New York. pp 19-59.

Kazahaya Y, Akimoto K, Otsuki S (1989). Subchronic methamphetamine treatment enhances methamphetamine- or cocaineinduced dopamine efflux in vivo. Biol Psychiatry 25: 903-912.

Kolta MG, Shreve P, De Souza V, Uretsky NJ (1985). Time course of the development of the enhanced behavioral and biochemical responses to amphetamine after pretreatment with amphetamine. Neuropharmacology 24: 823-829.

Paulson PE, Robinson TE (1995). Amphetamine-induced timedependent sensitization of dopamine neurotransmission in the dorsal and ventral striatum: a microdialysis study in behaving rats. Synapse 19: 56-65.

Robinson TE, Becker JB (1982). Behavioral sensitization is accompanied by an enhancement in amphetamine-stimulated dopamine release from striatal tissue in vitro. Eur J Pharmacol 85: 253-254.

Robinson TE, Becker JB (1986). Enduring changes in brain and behavior produced by chronic amphetamine administration: a review and evaluation of animal models of amphetamine psychosis. Brain Res Rev 11: 157-198.

Robinson TE, Berridge KC (2000). The psychology and neurobiology of addiction: an incentive-sensitization view. Addiction 95(Suppl. 2): S91-S117.

Robinson TE, Berridge KC (2003). Addiction. Ann Rev Psychol 54: 25-53.
Robinson TE, Kolb B (1997). Persistent structural modifications in nucleus accumbens and prefrontal cortex neurons produced by previous experience with amphetamine. J Neurosci 17: 84918497.

Robinson TE, Kolb B (1999a). Alterations in the morphology of dendrites and dendritic spines in the nucleus accumbens and prefrontal cortex following repeated treatment with amphetamine or cocaine. Eur J Neurosci 11: 1598-1604.

Robinson TE, Kolb B (1999b). Morphine alters the structure of neurons in the nucleus accumbens and neocortex of rats. Synapse 33: 160-162.

Segal DS, Geyer MA, Schuckit MA (1981). Stimulant-induced psychosis: an evaluation of animal models. Essays Neurochem Neuropharmacol 5: 95-129.

Smith AD, Bolam JP (1990). The neural network of the basal ganglia as revealed by the study of synaptic connections of identified neurons. Trends Neurosci 13: 259-265.

Vanderschuren LJ, Kalivas PW (2000). Alterations in dopaminergic and glutamatergic transmission in the induction and expression of behavioral sensitization: a critical review of preclinical studies. Psychopharmacology 151: 99-120.

Wolf ME (1998). The role of excitatory amino acids in behavioral sensitization to psychomotor stimulants. Prog Neurobiol 54: 679-720. 